\title{
Speech and Mild Cognitive Impairment detection
}

\author{
Charalambos Themistocleous ${ }^{1,2}$, Dimitrios Kokkinakis ${ }^{2,3}$ \\ ${ }^{1}$ Department of Neurology, Johns Hopkins University, USA \\ 2 Department of Swedish, University of Gothenburg, Sweden \\ ${ }^{3}$ Centre for Ageing and Health, University of Gothenburg, Sweden \\ https://doi.org/10.36505/ExLing-2019/10/0050/000412
}

\begin{abstract}
It is of great importance to detect objective markers that can enable the early and fast identification of individuals with Mild Cognitive Impairment (MCI) from healthy individuals to inform, patient care, family and treatment planning. Connected speech productions can offer such markers. This study analyses recordings from picture description tasks by Swedish individuals with MCI and healthy control individuals (HC) and shows that voice quality, periodicity, and speech rate distinguish individuals with MCI from HC.
\end{abstract}

Key words: Mild Cognitive Impairment, Alzheimer's disease, speech analysis

\section{Introduction}

Mild Cognitive Impairment (MCI) does not always lead to Alzheimer's Disease (AD) but in most cases it does (Mitchell, Shiri-Feshki 2009). The challenge is to discover subtle markers early on that can identify individuals with MCI. A number of studies show that speech and language can provide such early markers (Asgari et al. 2017, Dodge et al. 2015, Roark et al. 2011, Satt, et al. 2014). Most studies focused primarily on acoustic markers that distinguish linguistic categories (for other features see e.g., Fraser et al. 2016), such as vowel formant frequencies, segmental duration (long/short vowels), and intonation and showed that they can provide early cues for MCI. In this line, we have developed a Deep Neural Network that identifies individuals with MCI and healthy controls (HC) with high classification accuracy $(\mathrm{M}=83 \%)$ by using only a couple of acoustic features (i.e., vowel duration, formant frequencies, and F0) (Themistocleous et al. 2018). Nevertheless, more research is required on acoustic features that associate with non-linguistic properties. The aim of this study is to identify early markers of MCI from voice quality, speech rate, and periodicity.

\section{Methodology}

Thirty-two individuals with MCI and third-two HC individuals between 55 and 79 years old $(\mathrm{M}=69, \mathrm{SD}=6.4)$ participated in the study; these individuals did not differ with respect to age $[\mathrm{t}(52.72)=-1.8178, \mathrm{p}=\mathrm{n} . \mathrm{s}$. $]$ and gender $(\mathrm{W}=$ 1567.5, $\mathrm{p}=$ n.s.). Six selection criteria were followed: (i) participants should be

ExLing 2019: Proceedings of 10 $0^{\text {th }}$ International Conference of Experimental Linguistics, 25-27 September 2019, Lisbon, Portugal 
Swedish speakers; (ii) be able to read understand information about the study; (iii) provide written consent and not suffer from (iv) dyslexia and reading difficulties; (v) major depression, ongoing substance abuse; (vi) other psychiatric, neurological or brain-related conditions. Healthy control individuals had higher Mini-Mental State Exam (MMSE) score. Ethic approvals for the study were obtained by the local ethical committee review board (reference number: L091-99, 1999; T479-11, 2011); while the currently described study was approved by the local ethical committee decision 206-16, 2016. The recordings were conducted in two phases the first phase begun in late 2016 and the second in 2018. We have measured (i) the overall production of MCI vs. HC ("Speaking Time"); (ii) the articulation rate of these two groups; (iii) voice quality measures, namely the difference between the first harmonic and third amplitude (H1-A3); and measures of periodicity, namely the Cepstral Peak Prominence (CPP). Higher values of CPP correspond to greater periodicity. We report linear regression models. In the tables, (1) significant $b$-weights indicate, $a$ significant semi-partial correlation. (2) $b$ stands for the unstandardized regression weights; (3) $s r^{2}$ represents the semi-partial correlation squared; (4) $L L$ and $U L$ indicate the lower limit of confidence intervals and their upper limits. One star * indicates $\mathrm{p}<.05$; two stars $* *$ indicate $\mathrm{p}<.01$.

Table 1. Regression results for speaking time.

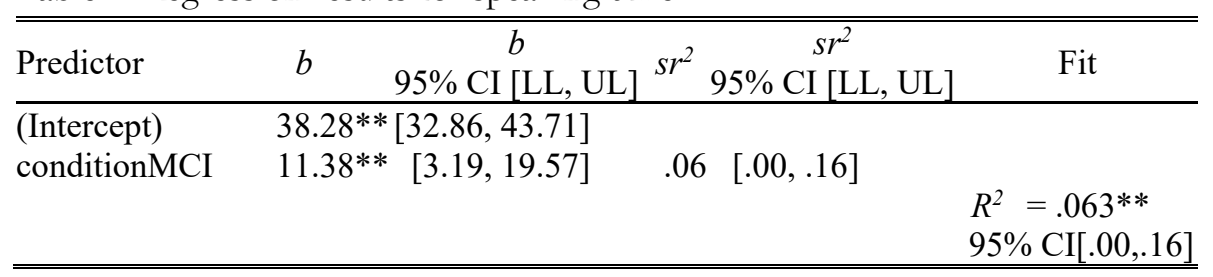

\section{Results}

MCI individuals produced slower speech; the effect resulted in statistically significant differences of individuals with MCI from HC (see Table 1).

Table 2. Regression results for articulation rate.

\begin{tabular}{|c|c|c|c|c|}
\hline Predictor & $b$ & $\begin{array}{c}b \\
95 \% \text { CI [LL, UL] }\end{array}$ & $\begin{array}{cc}s r^{2} & s r^{2} \\
& 95 \% \mathrm{CI}[\mathrm{LL}, \mathrm{UL}] \\
\end{array}$ & Fit \\
\hline (Intercept) & 65. & {$[58.46,72.48]$} & $06 \quad\left[\begin{array}{ll}00 & 16\end{array}\right.$ & \\
\hline ondtionilu & & & & $\begin{array}{l}R^{2}=.060^{* *} \\
95 \% \mathrm{CI}[.00, .16]\end{array}$ \\
\hline
\end{tabular}

Individuals with $\mathrm{MCI}$ had slower articulation rate than $\mathrm{HC}$ individuals (Table 2). Also, there were significant effects on voice quality effects. The H1-A3 was significantly different for individual factors of condition and gender and their interaction (Table 3). The results on CPP were significant for condition, gender, 
and their interaction. Especially, there was greater CPP for female HC speakers than female speakers with MCI (see Table 4).

Table 3 Regression results for $\mathrm{H} 1-\mathrm{A} 3$ (gender $\mathrm{M}=\mathrm{Male}$ ).

\begin{tabular}{lcccc}
\hline \hline Predictor & $b$ & $b$ & $s r^{2}$ & \multirow{2}{*}{ Fit } \\
\hline (Intercept) & $26.87 * *[25.14,28.59]$ & & \\
conditionMCI & $2.91 *$ & {$[0.52,5.29]$} & $.02[-.01, .05]$ & \\
genderM & $9.75^{*} *[6.11,13.39]$ & $.09[.03, .15]$ & \\
conditionMCI: & $-5.52 *[-10.06,-0.97]$ & $.02[-.01, .05]$ & \\
genderM & & & & $R^{2}=.137 * *$ \\
& & & $95 \%$ CI $[.06, .21]$ \\
\hline \hline
\end{tabular}

Table 4 Regression results for CPP (gender M=Male).

\begin{tabular}{lcccc}
\hline \hline Predictor & $b$ & $b$ & $s r^{2}$ & \multirow{2}{*}{ Fit } \\
\hline (Intercept) & $71.12^{* *}[70.59,71.66]$ & & \\
conditionMCI & $-1.18^{* *}[-1.92,-0.44]$ & $.03[-.01, .07]$ & \\
genderM & $-1.96^{* *}[-3.08,-0.83]$ & $.04[-.00, .08]$ & \\
conditionMCI: & $2.05 * *[0.64,3.46]$ & $.03[-.01, .07]$ & \\
genderM & & & $R^{2}=.057 * *$ \\
& & & $95 \% \mathrm{CI}[.01, .11]$ \\
\hline \hline
\end{tabular}

\section{Discussion}

This study analysed acoustic productions from recordings of a picture description task. Overall, individuals with MCI produce speech which is slower than the speech of HC individuals. Earlier studies suggest that MCI individuals produce longer pauses and longer segmental duration (see also Slegers et al. 2018). Our finding may suggest that longer vowel duration and longer pause duration can be attributed to the overall slower speech. Individuals with MCI differ from HCs on voice quality as well. These differences were shown on the difference between the first harmonic and third amplitude that associates with phonation Esposito (2010). Finally, the periodicity of speech in individuals with $\mathrm{MCI}$ and $\mathrm{HC}$ was found to differ with respect to CPP. Especially, there was lower CPP in female individuals with MCI than in female and male HC individuals, but the effects were less clear with respect to male individuals. Acoustic markers such as vowel formant frequencies that distinguish vowel quality, the fundamental frequency, which is the main acoustic correlate of intonation, segmental duration, etc. were studied extensively. These findings show that non-linguistic markers of speech production, such as speech periodicity, speech rate, and measures of voice quality can be employed to 
identify individuals with MCI from HC individuals. In our future research, we plan to integrate linguistic and non-linguistic acoustic properties elicited automatically from speech signals to identify individuals with MCI from HC individuals.

\section{Acknowledgements}

This research has been funded by Riksbankens Jubileumsfond - The Swedish Foundation for Humanities and Social Sciences (NHS 14-1761:1).

\section{References}

Asgari, M., Kaye, J., Dodge, H. 2017. Predicting mild cognitive impairment from spontaneous spoken utterances. Alzheimer's and Dementia: Translational Research \& Clinical Interventions, 3, 219-228.

Dodge, H., Mattek, N., Gregor, M., Bowman, M., Seelye, A., Ybarra, O., Kaye, J. 2015. Social markers of mild cognitive impairment. Current Alzheimer Research, 12, 513 519.

Esposito, C.M. 2010. The effects of linguistic experience on the perception of phonation. Journal of Phonetics 38, 306-316.

Fraser K.C., Meltzer J.A., Graham N.L., Leonard C., Hirst G., Black S.E., Rochon E. 2014. Automated classification of primary progressive aphasia subtypes from narrative speech transcripts. Cortex 55, 43-60.

Kokkinakis, D., Lundholm Fors, K., Fraser, K., Eckerström, M., Horn, G., Themistocleous, C. 2019. A multifaceted corpus for the study of cognitive decline in a Swedish population. In CLARe4. Helsinki, Finland.

Mitchell, A.J., Shiri-Feshki, M. 2009. Rate of progression of mild cognitive impairment to dementia - meta-analysis of 41 robust inception cohort studies. Acta Psychiatrica Scandinavica, 119: 252-265.

Roark, B., Mitchell, M., Hosom, J.P., Hollingshead, K., Kaye, J. 2011. Spoken language derived measures for detecting mild cognitive impairment. IEEE transactions on audio, speech, and language processing, 19(7), 2081-2090.

Satt, A., Hoory, R., König, A., Aalten, P.,Robert, P.H. 2014. Speech-based automatic and robust detection of very early dementia. In Fifteenth Annual Conference of the International Speech Communication Association.

Slegers, A., Filiou, R.P., Montembeault, M., Brambati, S. M. 2018. Connected Speech Features from Picture Description in Alzheimer's Disease: A Systematic Review. Journal of Alzheimer's Disease 65: 519-542.

Themistocleous C., Eckerström M., Kokkinakis, D. 2018. Identification of Mild Cognitive Impairment from Speech in Swedish using Deep Sequential Neural Networks. Frontiers in Neurology 9: 975. 